\title{
A RELATION BETWEEN THE COEFFICIENTS IN THE RECURRENCE FORMULA AND THE SPECTRAL FUNCTION FOR ORTHOGONAL POLYNOMIALS
}

BY

JEFFREY S. GERONIMO

\begin{abstract}
A relation is found between the rate of convergence of the coefficients in the recurrence formula for polynomials orthogonal on a segment of the real line and certain properties of the spectral function. The techniques of Banach algebras and scattering theory are used. The close connection between polynomials orthogonal on the unit circle and polynomials orthogonal on the real line is exploited.
\end{abstract}

1. Introduction. Suppose that one is given a nondecreasing spectral function $\rho(\lambda)$ with infinitely many points of increase on a segment $[a, b]$ of the real line such that

$$
s_{n}=\int \lambda^{n} d \rho(\lambda)
$$

exist for all $n$. It is well known that one can construct a set of polynomials [9] $\{p(\lambda, n)\}$, orthonormal with respect to $d \rho(\lambda)$ on the interval $[a, b]$ and that these polynomials satisfy the following three-term recurrence formula,

$$
\begin{aligned}
a(n+1) p(\lambda, n+1)+b(n) p(\lambda, n)+a(n) p(\lambda, n-1) & =\lambda p(\lambda, n), \\
n & =1,2,3, \ldots
\end{aligned}
$$

Here

$$
a(n)=\int_{a}^{b} \lambda p(\lambda, n) p(\lambda, n-1) d \rho(\lambda), \quad n=1,2, \ldots
$$

and

$$
b(n)=\int_{a}^{b} \lambda p(\lambda, n)^{2} d \rho(\lambda), \quad n=0,1,2, \ldots
$$

A question that comes to mind is, given the coefficients in the three-term recurrence formula, can one find a spectral function. This problem is an old one and it is a famous result of J. Favard's [2], [3] that if $p(\lambda,-1)=0, p(\lambda, 0)=K(0)$ $>0, a(n)>0, n>0$, and $b(n), n>0$, is real, then the polynomials $\{p(\lambda, n)\}$ constructed from (1.2) are orthogonal with respect to some spectral function $\rho(\lambda)$ which may not be unique. In this paper the same problem is considered except that stronger conditions are imposed on the coefficients in the recurrence formula. More precisely, let

$$
v(n)>1
$$

Received by the editors July 26, 1978 and, in revised form, March 6, 1979.

AMS (MOS) subject classifications (1970). Primary 42A52. 
be an even function of $\boldsymbol{n}$ with the following properties:

$$
\begin{aligned}
& v(n) \leqslant v(n+1), \quad n \geqslant 0, \\
& v(n) \leqslant v(m) v(n-m), \quad n, m \geqslant 0,
\end{aligned}
$$

and

$$
\lim \sup (v(n))^{1 / n}=1, \quad n \rightarrow \infty .
$$

The following theorem is the main result of the paper.

THEOREM 1. Let $\rho(\lambda)$ be a bounded nondecreasing, absolutely continuous function on the real interval $[a, b]$ with

$$
d \rho(\lambda) \approx \sigma(\theta) d \lambda_{j} \quad \text { where } \lambda \text { is related to } \theta \text { in (1.10). }
$$

Furthermore let $\sigma(\theta) / \sin \theta=\sigma(-\theta) / \sin (-\theta)$ and $\ln (\sigma(\theta) / \sin \theta)$ have an absolutely convergent Fourier series, then

$$
\sum_{n=0}^{\infty} n v(2 n)\left\{\left|1-\frac{a(n)^{2}}{a(\infty)^{2}}\right|+|B(n-1)|\right\}<\infty
$$

if and only if

$$
\sum_{n=-\infty}^{\infty}|n| v(n)|q(n)-q(n+2)|<\infty
$$

Here

$$
\begin{gathered}
\frac{\sigma(\theta)}{\sin \theta}=\sum_{n=-\infty}^{\infty} q(m) e^{i m \theta}, \\
\lambda=\left(\frac{b-a}{2}\right) \cos \theta+\left(\frac{b+a}{2}\right), \quad 0 \leqslant \theta \leqslant \pi, \\
\lim _{n \rightarrow \infty} a(n)=a(\infty)>0, \quad \lim _{n \rightarrow \infty} b(n)=b(\infty), \quad b(n) \text { real, }
\end{gathered}
$$

and

$$
B(n)=(b(n)-b(\infty)) / a(\infty) .
$$

The paper proceeds as follows; in §II the theory of orthogonal polynomials is briefly reviewed to develop the equations needed in the proof. In particular, a new set of two-two term recurrence formulas satisfied by polynomials orthogonal on the real line is exhibited. Next (in §III) a close connection is established between the solution of one of the above recurrence formulas and polynomials orthogonal on the unit circle. $\S I V$ contains the proof of Theorem 1. The proof of sufficiency is done in two ways. One way uses the close connection between polynomials orthogonal on the unit circle and those orthogonal on the real line. The other way (exhibited in Appendix A) uses the techniques of inverse scattering theory, in particular, the discrete analog of the Marchenko equation.

2. Preliminaries. If (1.11) holds then the polynomials satisfying (1.1) also satisfy the following set of recurrence formulas.

$$
\begin{aligned}
p(\lambda, n)=\frac{a(\infty)}{a(n)}[(Z-B(n-1)) p(\lambda, n-1)+\psi(Z, n-1) / Z], & \\
n & =1,2, \ldots,
\end{aligned}
$$


and

$$
\begin{array}{r}
\psi(Z, n)=\frac{a(\infty)}{a(n)}\left[\psi(Z, n-1) / Z+\left\{\left(1-\frac{a(n)^{2}}{a(\infty)^{2}}\right) Z-B(n-1)\right\} p(\lambda, n-1)\right] \\
n=1,2, \ldots
\end{array}
$$

$B(n)$ is given in (1.12) and $\lambda=a(\infty)(Z+1 / Z)+b(\infty)$. For initial conditions one takes

$$
p(\lambda, 0)=\psi(Z, 0)=K(0)>0 .
$$

Considering $p(\lambda, n)$ and $\psi(Z, n)$ as two components of a function $\Phi$ defined by

$$
\Phi(Z, n)=\left(\begin{array}{c}
p(\lambda, n) \\
\psi(Z, n)
\end{array}\right) .
$$

Equations (2.1) and (2.3) can be condensed to

$$
\Phi(Z, n)=C(n) \Phi(Z, n-1)
$$

where

$$
C(n)=\frac{a(\infty)}{a(n)}\left[\begin{array}{cc}
Z-B(n-1), & 1 / Z \\
\left\{\left(1-\frac{a(n)^{2}}{a(\infty)^{2}}\right) Z-B(n-1)\right\}, & 1 / Z
\end{array}\right]
$$

Two other useful solutions [4] of (2.5) are

$$
\Phi_{+}(Z, n)=\left(\begin{array}{l}
p_{+}(Z, n) \\
\psi_{+}(Z, n)
\end{array}\right)
$$

and

$$
\Phi_{-}(Z, n)=\left(\begin{array}{l}
p_{-}(Z, n) \\
\psi_{-}(Z, n)
\end{array}\right)
$$

satisfying the following boundary conditions:

$$
\begin{aligned}
\lim _{n \rightarrow \infty}\left|p_{ \pm}(Z, n)-Z^{ \pm n}\right|=0, & |Z| \leqslant 1, \\
\lim _{n \rightarrow \infty}\left|\psi_{+}(Z, n)\right|=0, & |Z|<1,
\end{aligned}
$$

and

$$
\lim _{n \rightarrow \infty}\left|\psi_{-}(Z, n)-\left(1-Z^{2}\right) Z^{-n}\right|=0, \quad|Z|<1
$$

It can be shown [4] that $\Phi_{+}(Z, n)$ and $\Phi_{-}(Z, n)$ are linearly independent for $Z=e^{i \theta}, Z \neq \pm 1$ and that

$$
\begin{array}{r}
\Phi(Z, n)=\frac{K(0)}{a(\infty)(Z-1 / Z)}\left[f_{-}(Z) \Phi_{+}(Z, n)-f_{+}(Z) \Phi_{-}(Z, n)\right] \\
|Z|=1, Z \neq \pm 1,
\end{array}
$$


where

$$
f_{+}(Z)=\frac{a(\infty)}{K(0) z} \lim _{n \rightarrow \infty} Z^{n} \psi(z, n),
$$

and

$$
f_{-}(Z)=\overline{f_{+}(Z)}=f_{+}(1 / Z), \quad|Z|=1 .
$$

Note from (2.5) and (2.9),

$$
\overline{p_{+}(Z, n)}=p_{+}(1 / Z, n)=p_{-}(Z, n), \quad|Z|=1 .
$$

One can include polynomials of the second kind in this scheme by defining

$$
\Phi_{\alpha}(Z, n)=\left(\begin{array}{c}
Q(\lambda, n) \\
\psi_{\alpha}(Z, n)
\end{array}\right), \quad n>1,
$$

satisfying (2.5) with boundary conditions

$$
Q(\lambda, 1)=\psi_{\alpha}(Z, 1)=(a(1))^{-1} K(0) .
$$

Now it is possible to write [4]

$$
p_{+}(Z, n)=K(0)\left[f_{+\alpha}(Z) p(\lambda, n)-f_{+}(Z) Q(\lambda, n)\right], \quad n \geq 1,
$$

where

$$
f_{+\alpha}(Z)=\frac{a(\infty)}{K(0) Z} \lim _{n \rightarrow \infty} Z^{n} \psi_{\alpha}(Z, n)
$$

Equation (2.15) can be extended to $n=0$ by defining $Q(\lambda, 0)=0$.

To proceed further it is convenient at this point to introduce the techniques of Banach algebras. Let $A_{v}$ denote the class of functions integrable on $-\Pi<\theta<\Pi$ such that if $g$ is an element of $A_{v}$ then

$$
g(\theta)=\sum_{K=-\infty}^{\infty} g(K) e^{i K \theta},
$$

with

$$
\|g\|_{0}=\sum_{K=-\infty}^{\infty} v(K)|g(K)|<\infty,
$$

where the properties of $v(n)$ are listed in (1.4) and (1.5). Let $A_{v}^{+}$and $A_{v}^{-}$denote those functions in $A_{v}$ of the form

$$
g(\theta)=\sum_{K=0}^{\infty} g(K) e^{i K \theta}
$$

and

$$
h(\theta)=\sum_{K=-\infty}^{0} h(K) e^{i K \theta},
$$

respectively.

Let $\|g\|_{v}$ be the norm of $A_{v}, A_{v}^{+}$and $A_{v}^{-}$, then $A_{v}, A_{v}^{+}$and $A_{v}^{-}$are Banach algebras. $A$ will denote the Banach algebra where $v(n)=1$ for all $n$. It is obvious that

$$
A_{v} \subset \boldsymbol{A}
$$


Returning now to (2.5) the following holds.

LEMMA 2.1. Given the initial condition (2.3) and the coefficients $a(n)$ and $B(n)$ in (2.5), if (1.7) and (1.11) are satisfied then

$$
\lim _{n \rightarrow \infty}\left\|\frac{a(\infty)}{K(0)} \psi^{*}(Z, n)-Z f_{+}(Z)\right\|_{0}=0
$$

and

$$
p_{+}(Z, n) \in A^{+}
$$

Here

$$
\psi^{*}(Z, n)=Z^{n} \psi(z, n)
$$

The proof follows from a minor modification of a similar proof given in Appendix B of [4].

The consequences of (1.7) for the spectral function $\rho(\lambda)$ are as follows:

TheOREM 2.1. Given (2.3) and $a(n)$ and $B(n)$ in (2.5), if (1.7) and (1.11) are satisfied and

$$
Z f_{+}(Z) \neq 0 \text { for }|Z|<1
$$

then

$$
\begin{aligned}
& d \rho(\lambda) \simeq \sigma(\theta) d \lambda, \quad b(\infty)-2 a(\infty)<\lambda<b(\infty)+2 a(\infty), \\
&(0<\theta<\pi)
\end{aligned}
$$

with

$$
\sigma(\theta)=\frac{a(\infty) \sin \theta}{\pi K(0)^{2}\left|f_{+}(Z)\right|^{2}} \quad \lambda=a(\infty)\left(e^{i \theta}+e^{-i \theta}\right)+b(\infty), \quad Z=e^{i \theta},
$$

and $[b(\infty)-2 a(\infty), b(\infty)+2 a(\infty)]$ is the interval of orthogonality. Furthermore $\sigma(\theta)=-\sigma(-\theta)$, and

$$
\frac{\sigma(\theta)}{\sin \theta} \in A_{v}
$$

Proof. The proof of (2.26) and (2.27) are given in $\$ 3$ of [4]. Equation (2.28) is a consequence of Lemma 2.1 (2.25), (2.27) and the Wiener-Levy theorem.

Equation (1.7) also has the following important consequence for the Fourier coefficients of the derivative of $f_{+}(z)$.

THEOREM 2.2. Given (2.3) if (1.7) and (1.11) are satisfied then

$$
\left(1-Z^{-2}\right)\left(Z f_{+}(Z)\right)^{\prime} \in A_{v}, \quad Z=e^{i \theta} \text {. }
$$

Here the differentiation is with respect to $Z$.

Proof. Let us begin by noting that from the recurrence formulas and (2.5), $\psi^{*}(Z, n)$ and $p(\lambda, n)$ can be written as

$$
\psi^{*}(Z, n)=\sum_{i=0}^{2 n} K(2 n, i) Z^{i}
$$


and

$$
p(\lambda, n)=\sum_{i=0}^{2 n} \hat{K}(2 n, i) Z^{i-n} .
$$

From the recurrence formulas,

$$
K(2 n, 0)=K(0) \prod_{i=1}^{n} a(\infty) / a(i) \neq 0 .
$$

Therefore these equations can be written as

$$
\frac{\psi^{*}(Z, n)}{K(2 n, 0)}=1+\sum_{i=1}^{2 n} h(2 n, i) Z^{i}
$$

and

$$
\frac{p(\lambda, n)}{K(2 n, 0)}=\sum_{i=0}^{2 n} \hat{h}(2 n, i) Z^{i-n}
$$

where

$$
h(2 n, i)=K(2 n, i) / K(2 n, 0)
$$

and

$$
\hat{h}(2 n, i)=\hat{K}(2 n, i) / K(2 n, 0) \text {. }
$$

Letting $n \rightarrow \infty$ in (2.32), it follows from Lemma 2.1, (2.31a) and (2.2) (after multiplying by $Z^{n} / K(2 n, 0)$ and iterating downward) that

$$
\begin{aligned}
\frac{K(0) Z f_{+}(Z)^{1}}{a(\infty) K(\infty, 0)} & =1+\sum_{m=1}^{\infty} h(m) Z^{m} \\
& =1+\sum_{i=0}^{\infty}\left\{\left(1-\frac{a(i+1)^{2}}{a(\infty)^{2}}\right) Z^{2}-B(i) Z\right\} Z^{i} \frac{p(\lambda, i)}{K(2 i, 0)},
\end{aligned}
$$

where

$$
h(m)=h(\infty, m)
$$

Substituting (2.33) into (2.36) gives

$$
\begin{aligned}
& \frac{K(0) Z f_{+}(Z)}{a(\infty) K(\infty, 0)} \\
& \quad=1+\sum_{\gamma=0}^{\infty} \sum_{i=[(\gamma+1) / 2]}^{\infty}\left\{\left(1-\frac{a(i+1)^{2}}{a(\infty)^{2}}\right) Z^{\gamma+2}-B(i) Z^{\gamma+1}\right\} \hat{h}(2 i, \gamma),
\end{aligned}
$$

where $[b]$ means the largest integer less than or equal to $b$.

Multiplying (2.36) by $Z\left(1-Z^{-2}\right)$ and equating coefficients gives

\footnotetext{
'It is without loss of generality that $Z f_{+}(Z)$ is set equal to its Fourier series.
} 


$$
\begin{aligned}
& \nu(m)|h(m)-h(m+2)|<\nu(m) \sum_{i=[(m-1) / 2]}^{[(m+1) / 2]-1}\left|\left(1-\frac{a(i+1)^{2}}{a(\infty)^{2}}\right) \hat{h}(2 i, m-2)\right| \\
& +\nu(m) \sum_{i=[m / 2]}^{[(m+2) / 2]-1}|B(i) \hat{h}(2 i, m-1)| \\
& +\nu(m) \sum_{i=[(m+1) / 2]}^{\infty}\left|\left(1-\frac{a(i+1)^{2}}{a(\infty)^{2}}\right)(\hat{h}(2 i, m-2)-\hat{h}(2 i, m))\right| \\
& +\nu(m) \sum_{i=[(m+1) / 2]}^{\infty}|B(i)(\hat{h}(2 i, m-1)-\hat{h}(2 i, m+1))|, \quad m>1
\end{aligned}
$$

Letting $Z \rightarrow 1 / Z$ in (2.7), then subtracting it from the original equation yields

$$
\left(1-Z^{2}\right) 1 / Z p(\lambda, n-1)=1 / Z \psi(Z, n-1)-Z \psi(1 / Z, n-1) .
$$

Multiplying by $Z^{n} / K(2 n, 0)$ one finds from Lemma 2.1 that

$$
\begin{aligned}
\left\|\left(1-Z^{2}\right) Z^{n-1} \frac{p(\lambda, n-1)}{K(2 n-2,0)}\right\|<\left\|\frac{\psi^{*}(Z, n)}{K(2 n-2,0)}\right\|+\left\|\frac{Z^{2 n+2} \psi^{*}(1 / Z, n)}{K(2 n-2,0)}\right\| \\
<M<\infty \quad \text { for all } n, Z=e^{i \theta} .
\end{aligned}
$$

Therefore, from (2.33),

$$
\sum_{j=0}^{2 i+2}|\hat{h}(2 i, j-2)-\hat{h}(2 i, j)|<M<\infty \quad \text { for all } i,
$$

where

$$
\hat{h}(2 i, j) \equiv 0 \text { for } j<0 \text { and } j>2 i .
$$

Equations (2.42) and (2.43) imply that the Fourier coefficients are uniformly bounded for all $n$. To see this, note that from (2.42),

$$
|\hat{h}(2 i, K)-\hat{h}(2 i, K+2)|<\sum_{j=0}^{2 i+2}|\hat{h}(2 i, j-2)-\hat{h}(2 i, j)|<M
$$

The above equation and (2.43) imply

$$
\text { for all } i,-2<K<2 i \text {. }
$$

$$
|\hat{h}(2 i, K)|<2 M \text { for all } i, 0<K<2 i \text {. }
$$

Multiplying (2.39) by $m$, summing and using the properties of $v(m)$ one finds, using the above results,

$$
\begin{aligned}
\sum_{m=2}^{\infty} m v(m)|h(m)-h(m+2)| \\
<M \sum_{m=2}^{\infty} \sum_{i=[(m-1) / 2]}^{[(m+1) / 2]-1}(2 i+2) v(2 i+2)\left|1-\frac{a(i+1)^{2}}{a(\infty)^{2}}\right| \\
+M \sum_{m=2}^{\infty} \sum_{i=[m / 2]}^{[(m+2) / 2]-1}(2 i+2) v(2 i+2)|B(i)| \\
+M \sum_{i=1}^{\infty} 2 i v(2 i)\left\{\left|1-\frac{a(i)^{2}}{a(\infty)^{2}}\right|+|B(i-1)|\right\}<\infty .
\end{aligned}
$$


3. The properties of $\psi(Z, n)$ and $p_{+}(Z, n)$ and $f_{+\alpha}(Z)$. In this section the properties of $\psi(Z, n)$ are investigated. In particular, it will be shown that under certain circumstances $\{\psi(Z, n)\}$ are closely related to polynomials orthogonal on the unit circle.

THEOREM 3.1. Given (2.3), (1.7), (1.11) and (2.25) then $\sqrt{\pi a(\infty)} Z^{n} \psi(1 / Z, n)$ is a polynomial on the unit circle orthonormal with respect to the weight $\sigma(\theta) / \sin \theta$.

Proof. From Theorem 2.1, $\sigma(\theta) / \sin \theta$ can be constructed from (2.27). Now it is shown that

$$
\frac{1}{2 \pi} \int_{-\pi}^{\pi} Z^{-m} Z^{n} \psi(1 / Z, n) \frac{\sigma(\theta)}{\sin \theta} d \theta=0, \quad 0<m<2 n, Z=e^{i \theta}
$$

and

$$
\frac{1}{2 \pi} \int_{b-\pi}^{\pi} \pi a(\infty) \psi(Z, n) \psi(1 / Z, n) \frac{\sigma(\theta)}{\sin \theta} d \theta=1, \quad Z=e^{i \theta}
$$

This result is most easily proved by letting $Z \rightarrow 1 / Z$ in (2.10) then substituting the lower component and (2.27) into the above equations. For example, (3.1) becomes

$$
\begin{aligned}
& =\frac{1}{2 \pi} \int_{-\pi}^{\pi} \frac{Z^{n-m} \psi_{+}(1 / Z, n)}{(1 / Z-Z) f_{-}(Z) \frac{K(0) \pi}{a(\infty)}} d \theta \\
& -\frac{1}{2 \pi} \int_{-\pi}^{\pi} \frac{Z^{n-m} \psi_{-}(1 / Z, n)}{(1 / Z-Z) f_{+}(Z) \frac{K(0) \pi}{a(\infty)}} d \theta, \quad Z=e^{i \theta} .
\end{aligned}
$$

Now letting $\theta \rightarrow-\theta$ in the first integral and noting from the recurrence formulas that

$$
\begin{aligned}
Z f_{+}(Z) & =a(\infty) \prod_{i=1}^{\infty} \frac{a(\infty)}{a(i)}+O(Z), \\
\psi_{+}(Z, n) & =\prod_{i=n+1}^{\infty} \frac{a(\infty)}{a(i)} B(n) Z^{n+1}+O\left(Z^{n+2}\right),
\end{aligned}
$$

and

$$
\psi_{-}(1 / Z, n)=\prod_{i=n+1}^{\infty} \frac{a(\infty)}{a(i)}\left(1-1 / Z^{2}\right) Z^{n}+O\left(Z^{n-1}\right)
$$

gives the desired result. Equation (3.2) is proved in a similar fashion. Since the leading coefficient of $\sqrt{\pi a(\infty)} Z^{n} \psi(1 / Z, n)$ is positive the above results imply that $\sqrt{\pi a(\infty)} Z^{n} \psi(1 / Z, n)$ is a polynomial orthonormal on the unit circle.

Define

$$
\begin{array}{r}
F(Z)=\frac{1}{2 \pi} \int_{-\pi}^{\pi}\left(\frac{e^{i \theta}+Z}{e^{i \theta}-Z}\right) \frac{\sigma(\theta)}{\sin \theta} d \theta, \quad|Z|<1, \\
\hat{\psi}(Z, n)=\frac{\sqrt{a(\infty) \pi}}{2 \pi} \int_{\pi}^{\pi} \frac{\left(e^{i \theta}+Z\right)}{\left(e^{i \theta}-Z\right)}\left(w^{n} \psi(1 / w, n)-Z^{\prime n} \psi(1 / Z, n)\right) \frac{\sigma(\theta)}{\sin \theta} d \theta, \\
|Z|<1, w=e^{i \theta},
\end{array}
$$


and

$$
\frac{\sigma(n, \theta)}{\sin \theta}=\frac{1}{\pi a(\infty)} \frac{1}{\left|\psi^{*}(Z, n)\right|^{2}}=\sum_{m=-\infty}^{\infty} q(n, m) Z^{m}, \quad Z=e^{i \theta}
$$

LEMMA 3.1. Given (2.3), (1.7), (1.11), and (2.25), then

$$
q(n, m)=q(m), \quad|m|<2 n+3 .
$$

$q(n, m)$ is defined in (3.7) and $q(m)$ in (1.9).

Proof. It follows from Theorems 3.1 and 2.1 that $\sqrt{\pi a(\infty)} Z^{n} \psi(1 / Z, n)$ is a polynomial on the unit circle orthogonal with respect to a positive weight $\sigma(\theta) / \sin \theta$ and $F(Z)$ is analytic inside the unit circle and continuous on it. Therefore, from the theory of orthogonal polynomials on the unit circle [7], [9],

$$
Z^{n} \psi(1 / Z, n) \neq 0, \quad|Z|>1,
$$

and (3.7) is well defined. From (3.5) and (3.6),

$$
\begin{array}{r}
F(Z) \sqrt{a(\infty) \pi} \psi^{*}(Z, n)-\hat{\psi}^{*}(Z, n) \\
=\frac{Z^{2 n} \sqrt{a(\infty) \pi}}{2 \pi} \int_{-\pi}^{\pi}\left(1+2 \sum_{K=1}^{\infty} Z^{K} w^{-n-K}\right) \psi(w, n) \frac{\sigma(\theta)}{\sin \theta} d \theta, \\
w=e^{i \theta},|Z|<1,
\end{array}
$$

where

$$
\hat{\psi}^{*}(Z, n)=Z^{2 n} \overline{\hat{\psi}(Z, n)}, \quad|Z|=1 .
$$

Substituting (2.10) and (2.27) then using (3.4) yields

$$
F(Z) \sqrt{a(\infty) \pi} \psi^{*}(Z, n)-\hat{\psi}^{*}(Z, n)=O\left(Z^{2 n+3}\right), \quad|Z|<1 .
$$

Since $\psi^{*}(Z, n) \neq 0,|Z|<1$ (see (2.24) and (3.9)) the above equation implies $F(Z)$ has the same Fourier coefficients in $\left\{Z^{i}\right\}_{0}^{2 n+2}$ as $\hat{\psi}^{*}(Z, n) / \sqrt{a(\infty) \pi} \psi^{*}(Z, n)$. Now [7], [9],

$$
\sqrt{\pi a(\infty)}\left[\hat{\psi}(Z, n) \psi^{*}(Z, n)+Z^{n} \psi(1 / Z, n) \hat{\psi}^{*}(Z, n)\right]=2 Z^{2 n}, \quad|Z|=1,
$$

therefore

$$
\operatorname{Re}\left[\frac{\hat{\psi}^{*}(Z, n)}{\sqrt{\pi a(\infty)} \psi^{*}(Z, n)}\right]=\frac{1}{\pi a(\infty)\left|\psi^{*}(Z, n)\right|^{2}}, \quad|Z|=1 .
$$

Equations (3.11), (3.13) and the reality of $\sigma(\theta) / \sin \theta$ give the result.

Now let us investigate the properties of $p_{+}(Z, n)$ and $f_{+\alpha}(Z)$. Note that if (1.7), (1.11) and (2.3) are satisfied then a lemma similar to Lemma 2.1 holds [4] for $\psi_{\alpha}(Z, n)$ and $f_{+\alpha}(Z)$. If $(2.25)$ is also satisfied then the following is true.

LEMMA 3.2. Given (2.5) and (2.3), if (1.7), (1.11) and (2.25) are satisfied then

$$
f_{+\alpha}(Z)=f_{+}(Z) \int_{a}^{b} \frac{\sigma\left(\theta^{\prime}\right)}{\lambda-\lambda^{\prime}} d \lambda^{\prime}, \quad|Z|<1,
$$


and

$$
p_{+}(Z, n)=K(0) f_{+}(Z) \int_{a}^{b} \frac{p\left(\lambda^{\prime}, n\right) \sigma\left(\theta^{\prime}\right)}{\lambda-\lambda^{\prime}} d \lambda^{\prime}, \quad|Z|<1 .
$$

Proof. Solving for $\psi(Z, n-1) / Z$ in (2.1) then substituting the result into (2.4) yields

$$
\psi(Z, n)=p(\lambda, n)=\frac{a(n)}{a(\infty)} Z p(\lambda, n-1) .
$$

Since $\Phi_{\alpha}(Z, n)$ satisfies (2.5) one can also write

$$
\psi_{\alpha}(Z, n)=Q(\lambda, n)-\frac{a(n)}{a(\infty)} Z Q(\lambda, n-1), \quad n>1
$$

Since $Q(\lambda, n)$ is a polynomial of the second kind,

$$
Q(\lambda, n)=\int_{a}^{b}\left(\frac{p(\lambda, n)-p\left(\lambda^{\prime}, n\right)}{\lambda-\lambda^{\prime}}\right) \sigma\left(\theta^{\prime}\right) d \lambda^{\prime}, \quad n>1
$$

Therefore

$$
\begin{aligned}
\psi_{\alpha}(Z, n)= & \psi(Z, n) \int_{a}^{b} \frac{\sigma\left(\theta^{\prime}\right) d \lambda^{\prime}}{\lambda-\lambda^{\prime}} \\
& -\int_{a}^{b} \frac{\left(p\left(\lambda^{\prime}, n\right)-Z(a(n) / a(\infty)) p\left(\lambda^{\prime}, n-1\right)\right)}{\lambda-\lambda^{\prime}} \sigma\left(\theta^{\prime}\right) d \lambda^{\prime}
\end{aligned}
$$

The integral

$$
\begin{aligned}
\int_{a}^{b} \frac{p\left(\lambda^{\prime}, n\right)}{\lambda-\lambda^{\prime}} \sigma\left(\theta^{\prime}\right) d \lambda^{\prime} & =\frac{-1}{2 i} \int_{-\pi}^{\pi} p\left(\lambda^{\prime}, n\right) \sigma\left(\theta^{\prime}\right)\left(\frac{e^{i \theta^{\prime}}+Z}{e^{i \theta^{\prime}}-Z}\right) d \theta^{\prime} \\
& =\frac{-1}{2 i} \int_{-\pi}^{\pi} \sin \theta^{\prime} p\left(\lambda^{\prime}, n\right) \frac{\sigma\left(\theta^{\prime}\right)}{\sin \theta^{\prime}}\left(\frac{e^{i \theta^{\prime}}+Z}{e^{i \theta^{\prime}}-Z}\right) d \theta^{\prime},
\end{aligned}
$$

where the property $\sigma(\theta)=-\sigma(-\theta)$ has been used. Here

$$
\lambda^{\prime}=2 a(\infty) \cos \theta^{\prime}+b(\infty)
$$

and

$$
\lambda=a(\infty)(Z+1 / Z)+b(\infty) .
$$

Therefore it follows from Theorem 2.1 and (2.41) that the above integral is bounded for $|Z|<1(\lambda>1)$. Multiplying through by $Z^{n}$ then letting $n \rightarrow \infty$ using Lemma 2.1 and (2.16) yields

$$
f_{+\alpha}(Z)=f_{+}(Z) \int_{a}^{b} \frac{\sigma\left(\theta^{\prime}\right)}{\lambda-\lambda^{\prime}} d \lambda^{\prime}, \quad|Z|<1 .
$$

Since

$$
\int_{a}^{b} \frac{\sigma\left(\theta^{\prime}\right)}{\lambda-\lambda^{\prime}} d \lambda^{\prime}=\frac{-1}{2 i} \int_{-\pi}^{\pi} \sigma\left(\theta^{\prime}\right)\left(\frac{e^{i \theta^{\prime}}+Z}{e^{i \theta^{\prime}}-Z}\right) d \theta^{\prime},
$$

and from Theorem 2.1, $\sigma(\theta)$ is an element of $A,(3.23)$ is true for $|Z|=1$. Substituting (3.23) into (2.15) gives

$$
p_{+}(Z, n)=K(0) f_{+}(Z) \int_{a}^{b} \frac{p\left(\lambda^{\prime}, n\right) \sigma\left(\theta^{\prime}\right)}{\lambda-\lambda^{\prime}} d \theta^{\prime}, \quad|Z|<1, n>1 .
$$


In all of the previous theorems and lemmas it has been assumed that (1.7) held. Now we begin with assumptions on the weight.

TheOREM 3.2. Assume $\rho(\lambda)$ is a bounded nondecreasing function on the real interval $[a, b]$ with an infinite number of points of increase. Furthermore

$$
\rho(\lambda) \approx \sigma(\theta) d \lambda, \quad \lambda=\frac{b-a}{2} \cos \theta+\frac{a+b}{2}, \quad 0<\theta<\pi .
$$

with

$$
\frac{\sigma(\theta)}{\sin \theta}=\frac{\sigma(-\theta)}{\sin (-\theta)}
$$

and

$$
\ln \frac{\sigma(\theta)}{\sin \theta} \in A \text {. }
$$

Then $a(\infty)$ exists and is positive, $b(\infty)$ exists, and $f_{+}(Z)$ can be uniquely constructed such that

$$
\begin{gathered}
\frac{\sigma(\theta)}{\sin \theta}=\frac{a(\infty)}{\pi K(0)^{2}\left|Z f_{+}(Z)\right|^{2}}, \quad Z=e^{i \theta}, \\
Z f_{+}(Z) \neq 0, \quad|Z|<1, \\
Z f_{+}(Z) \in A^{+}, \quad Z=e^{i \theta},
\end{gathered}
$$

and

$$
\left.Z f_{+}(Z)\right|_{z=0}>0
$$

Furthermore (3.1), (3.2), and (3.8) hold and

$$
p_{+}(Z, n) \in A^{+} \text {. }
$$

In (3.29),

$$
K(0)^{2}=\left(\int_{a}^{b} \sigma(\theta) d \lambda\right)^{-1}>0 .
$$

Proof. $\sigma(\theta) / \sin \theta$ can be thought of as a weight function defined on the unit circle and one can uniquely construct a set of polynomials $\{\phi(Z, n)\}$ defined on the unit circle orthonormal with respect to $\sigma(\theta) / \sin \theta$. Baxter [1] has shown that (3.28) implies that

$$
\phi^{*}(Z, n) \in A^{+} \text {for all } n
$$

and $\left\{\phi^{*}(z, n)\right\}$ is a Cauchy sequence in $A^{+}$. Here

$$
\phi^{*}(Z, n)=Z^{n} \phi(1 / Z, n), \quad Z=e^{i \theta} .
$$

The polynomials $\{p(\lambda, n)\}$ orthonormal with respect to $\sigma(\lambda)$ can [9] be written as

$$
\begin{aligned}
\left(Z-Z^{-1}\right) p(\lambda, n) & =c(n)\left[Z^{-n-1} \phi(Z, 2 n+2)=Z^{n+1} \phi(1 / Z, 2 n+2)\right] \\
c(n) & =\left(\frac{2}{\pi}\right)^{1 / 2}\left(1-\frac{\theta(0,2 n+2)^{-1 / 2}}{k(2 n+2)}\left(\frac{2}{b-a}\right)^{1 / 2},\right.
\end{aligned}
$$


where $\lambda$ is written as in (3.27) and $k(2 n+2)$ is the leading coefficient of $\phi(z, 2 n+$ 2). Since $|\phi(0,2 n+2) / k(2 n+2)|<1$, and $\phi(0,2 n+2) \rightarrow 0$ uniformly as $n \rightarrow \infty$, equation (3.37) implies

$$
\left(Z-Z^{-1}\right) p(\lambda, n) \in A, \quad Z=e^{i \theta} .
$$

Therefore, since $\ln \sigma(\theta) / \sin \theta \in A$ implies $\sigma(\theta) / \sin \theta \in A$,

$$
\begin{array}{r}
a(\infty)=\lim _{n \rightarrow \infty} a(n)=\lim _{n \rightarrow \infty} \int_{a}^{b} \lambda p(\lambda, n) p(\lambda, n-1) \sigma(\theta) d \lambda \\
=\lim _{n \rightarrow \infty}\left(\frac{b-a}{2}\right) \int_{0}^{\pi}\left(\left(\frac{b-a}{2}\right) \cos \theta+\left(\frac{b+a}{2}\right)\right) \\
\cdot p(\theta, n) p(\theta, n-1) \frac{\sigma(\theta)}{\sin \theta} \sin ^{2} \theta d \theta .
\end{array}
$$

The existence of $b(\infty)$ follows from (1.3) and an argument similar to the one given above. Equations (3.29)-(3.32) are consequences of the Wiener-Levy theorem [1], [4] and $f_{+}(Z)$ may be explicitly constructed from $\sigma(\theta) / \sin \theta$ using a modification of the Poisson-Integral formula [4].

One now constructs

$$
p_{+}(Z, n) \in A^{+}, \quad n>1,
$$

from (3.25). It is easy to see that $p_{+}(Z, n)$ satisfies the recurrence formulas and it can be shown [5] using (3.37) that the appropriate boundary condition (2.9) is also satisfied. $p_{-}(Z, n)$ is formed using (2.12a). One can now derive (3.4) from the recurrence formulas and (3.1), (3.2) and (3.8) follow.

4. Proof of Theorem 1. The following will now be proved.

THEOREM 1. Let $\rho(\lambda)$ be a bounded nondecreasing, absolutely continuous function on the real interval $[a, b]$ with

$$
d \rho(\lambda) \approx \sigma(\theta) d \lambda, \quad \lambda=\left(\frac{b-a}{2}\right) \cos \theta+\frac{b+a}{2}, \quad 0<\theta<\pi .
$$

Furthermore, let $\sigma(\theta) / \sin \theta=\sigma(-\theta) / \sin (-\theta)$ and $\ln (\sigma(\theta) / \sin \theta) \in A$, then

$$
\sum_{n=1}^{\infty} n v(2 n)\left\{\left|\left(1-\frac{a(n)^{2}}{a(\infty)^{2}}\right)\right|+|B(n-1)|\right\}<\infty
$$

if and only if

$$
\sum_{n=-\infty}^{\infty}|n| v(n)|q(n)-q(n+2)|<\infty
$$

Here

$$
\frac{\sigma(\theta)}{\sin \theta}=\sum_{m=-\infty}^{\infty} q(m) e^{i m \theta}
$$

(It is without loss of generality that $\sigma(\theta) / \sin \theta$ is set equal to its Fourier series and $v(n)$ is defined in (1.4) and (1.5).)

Proof. Note that the hypotheses imply $a(n)>0$ for all $n$ and we take

$$
K(0)=\left(\int_{b}^{a} \sigma(\theta) d \lambda\right)^{-1 / 2}>0 .
$$


The above assumptions allow us to call upon Theorem 3.2, giving

$$
\frac{\sigma(\theta)}{\sin \theta}=\sum_{m=-\infty}^{\infty} q(m) e^{i m \theta}=\frac{a(\infty)}{\pi K(0)^{2}\left|Z f_{+}(Z)\right|^{2}}, \quad Z=e^{i \theta}
$$

with

$$
Z f_{+}(Z) \neq 0, \quad|Z|<1 \text { and } e^{i \theta} f_{+}\left(e^{i \theta}\right) \in A^{+} .
$$

If (1.7) holds then Lemma 2.1 implies

$$
Z f_{+}(Z) \in A_{0}^{+}, \quad Z=e^{i \theta},
$$

and from (4.1) and the Wiener-Levy theorem,

$$
\frac{\sigma(\theta)}{\sin \theta} \in A_{v} \text {. }
$$

Differentiating ${ }^{2}$ both sides of (4.1) with respect to $\theta$, then multiplying by, $\left(1-e^{-2 i \theta}\right)$ yields

$$
\begin{aligned}
\sum_{m=-\infty}^{\infty} m(q(m) & -q(m+2)) e^{i m \theta}=2 e^{-2 i \theta} \sum_{m=-\infty}^{\infty} i q(m) e^{i m \theta} \\
+ & \frac{a(\infty)}{\pi K(0)^{2}}\left[\frac{\left(1-e^{-2 i \theta}\right)\left(e^{i \theta} f_{+}\left(e^{i \theta}\right)\right)^{\prime}}{\left|e^{i \theta} f_{+}\left(e^{i \theta}\right)\right|^{2} e^{i \theta} f_{+}\left(e^{i \theta}\right)}+\frac{\left(1-e^{-2 i \theta}\right)\left(e^{-i \theta} f_{+}\left(e^{-i \theta}\right)\right)^{\prime}}{\left|e^{i \theta} f_{+}\left(e^{i \theta}\right)\right|^{2}\left(e^{-i \theta} f_{+}\left(e^{-i \theta}\right)\right)}\right] .
\end{aligned}
$$

Equations (4.1)-(4.4), the Wiener-Levy theorem and Theorem 2.2 show that the right hand side of (4.5) is an element of $A_{v}$ thus giving (1.8).

To prove sufficiency note that $\ln (\sigma(\theta) / \sin \theta) \in A$ and (1.8) imply $\ln (\sigma(\theta) / \sin \theta)$ $\in A_{v}$. Calling upon Theorem 3.2 we see that $Z^{n} \psi(1 / Z, n)$ is a polynomial on the unit circle orthogonal with respect to $\sigma(\theta) / \sin \theta$ and Baxter [1] has shown that $\ln (\sigma(\theta) / \sin \theta) \in A_{v}$ implies

$$
\psi^{*}(Z, n) \rightarrow \frac{K(0)}{a(\infty)} Z f_{+}(Z) \text { in } A_{v} .
$$

Since $\psi^{*}(Z, n) \neq 0$ for $|Z|<1$ we define

$$
\frac{\sigma(n, \theta)}{\sin \theta}=\sum_{m=-\infty}^{\infty} q(n, m) Z^{m}=\frac{1}{\pi a(\infty)} \frac{1}{\left|\psi^{*}(Z, n)\right|^{2}} \in A_{v}, \quad Z=e^{i \theta}
$$

Differentiating the above equation with respect to $\theta$ and multiplying by (1 $Z^{-2}$ ) yields

$$
\begin{gathered}
\frac{1}{\pi a(\infty)}\left[\frac{\left(1-Z^{-2}\right) \psi^{*}(Z, n)^{\prime}}{\left|\psi^{*}(Z, n)\right|^{2} \psi^{*}(Z, n)}+\frac{\left(1-Z^{-2}\right) \psi^{*}(1 / Z, n)^{\prime}}{\left|\psi^{*}(Z, n)\right|^{2} \psi^{*}(1 / Z, n)}\right] \\
=\sum_{m=-\infty}^{\infty} i m(q(n, m)-q(n, m+2)) Z^{m} \\
\quad-2 \sum_{m=-\infty}^{\infty} i q(n, m+2) Z^{m}, \quad Z=e^{i \theta}
\end{gathered}
$$

\footnotetext{
${ }^{2}$ This was suggested to me by Professor P. G. Nevai.
} 
Multiplying by $C \psi^{*}(Z, n)^{2}$ where

$$
C=\pi a(\infty)
$$

and setting

$$
\psi^{*}(Z, n)^{2}=\sum_{i=0}^{4 n} C(n, i) Z^{i}
$$

yields

$$
\begin{aligned}
\left(1-Z^{-2}\right) & \frac{\psi^{*}(Z, n)^{\prime} / K(2 n, 0)}{\psi^{*}(1 / Z, n) / K(2 n, 0)}+\left(1-Z^{-2}\right) \frac{\psi^{*}(1 / Z, n)^{\prime} \psi^{*}(Z, n)}{\psi^{*}(1 / Z, n)} \\
= & C i \sum_{m=-\infty}^{\infty} Z^{m} \sum_{i=0}^{4 n} C(n, i)(m-i)(q(n, m-i)-q(n, m-i+2)) \\
& -2 C i \sum_{m=-\infty}^{\infty} Z^{m} \sum_{i=0}^{4 n} q(n, m-i+2) C(n, i), \quad Z=e^{i \theta}
\end{aligned}
$$

where $K(2 n, 0)$ is defined in (2.30). Since $\psi^{*}(Z, n) \neq 0$ for $|Z|<1$,

$$
\frac{K(2 n, 0)}{\psi^{*}(1 / Z, n)}=\sum_{K=0}^{\infty} \gamma(n, K) Z^{-K}
$$

with

$$
\sum|\gamma(n, K)|<\infty
$$

and from (2.32),

$$
\gamma(n, 0)=1 \text {. }
$$

Equating coefficients of $Z^{2 n}$ using (2.32) gives

$$
\begin{aligned}
& 2 n v(2 n)|h(2 n, 2 n)| \\
& =C v(2 n) \mid \sum_{i=0}^{4 n} C(n, i)((2 n-i)(q(n, 2 n-i)-q(n, 2 n-i+2)) \\
& \quad-2 q(n, 2 n-i+2)) \mid .
\end{aligned}
$$

From Theorem 3.2,

$$
q(n, m)=q(m), \quad|m|<2 n+2 .
$$

Therefore,

$$
\begin{aligned}
& 2 n v(2 n)|h(2 n, 2 n)| \\
& =C v(2 n) \mid \sum_{i=0}^{4 n} C(n, i)((2 n-i)(q(2 n-i)-q(2 n-i+2)) \\
& \quad-2 q(n, 2 n-i+2)) \mid .
\end{aligned}
$$


Summing on $n$ and using the properties of $v(n)((1.4)$ and (1.5)) gives

$$
\begin{aligned}
& 2 \sum_{n=2}^{\infty} n v(2 n)|h(2 n, 2 n)| \\
& <C \sum_{n=2}^{\infty}|2 n-i| v(2 n-i)|q(2 n-i)-q(2 n-i+2)| \sum_{i=0}^{4 n} v(i)|C(n, i)| \\
& \quad+2 C \sum_{n=2}^{\infty} v(2 n-i)|q(2 n-i+2)| \sum_{i=0}^{4 n} v(i)|C(n, i)| .
\end{aligned}
$$

Since $\sigma(\theta) / \sin \theta \in A_{v}$ and $(a(\infty) / K(0)) \psi^{*}(Z, n) \rightarrow Z f_{+}(Z)$ in $A_{v}$ the right-hand side of (4.17) is bounded. Multiplying (2.2) by $Z^{n}$, substituting in (2.32) and (2.33) then equating coefficients of $Z^{m}$ yields

$$
\begin{aligned}
h(2 n+2, m)= & h(2 n, m)+\left\{1-\frac{a(n)^{2}}{a(\infty)^{2}}\right\} \hat{h}(2 n, m-2) \\
& +B(m-1) \hat{h}(2 n, m-1) .
\end{aligned}
$$

Note that $\hat{h}(2 n, 2 n)=\hat{h}(2 n, 0)=1$ and $h(2 n, m)=\hat{h}(2 n, m)=0$ for $m>2 n$. Thus setting $m=2 n+2$ in (4.18) yields

$$
h(2 n+2,2 n+2)=\left\{1-\frac{a(n)^{2}}{a(\infty)^{2}}\right\} .
$$

Substituting the above equation into (4.17) and using the monotonicity property of $v(n)$ gives

$$
\sum_{n=1}^{\infty} n v(2 n)\left|1-\frac{a(n)^{2}}{a(\infty)^{2}}\right|<\infty
$$

To show that the $B(n)$ 's converge at a similar rate return to (4.11) and equate coefficients of $Z^{2 n-1}$, thus

$$
\begin{aligned}
v(2 n)(2 n-1)|h(2 n, 2 n-1)|< & 2 n v(2 n)|h(2 n, 2 n)\{h(2 n, 1)+\gamma(n, 1)\}| \\
& +C v(2 n) \sum_{i=1}^{4 n}|C(n, i)|(2 n-1-i) \\
& \cdot|q(n, 2 n-i-1)-q(n, 2 n-i+1)| \\
& +2 C v(2 n) \sum_{i=0}^{4 n}|q(n, 2 n-i+1)||C(n, i)| .
\end{aligned}
$$

Setting $m=2 n+1$ in (4.18), then substituting the result into (4.21), summing on $n$ and using the fact that $h(n, 1), \hat{h}(n, 1)$ and $\gamma(n, 1)$ are bounded for all $n$ yields the desired result.

An alternative proof of sufficiency using the discrete analog of the Marchenko equation is given in Appendix $\mathbf{A}$.

5. Conclusions. In this paper the coefficients in the recurrence relation have been assumed to converge at a particular rate. Consequences for the spectral function, of the above assumptions, have been found that are necessary and sufficient. 
One of the assumptions of Theorem 1 is that the spectral function be of the form $d \rho(\lambda)=\sigma(\theta) d \lambda$ with $\ln (\sigma(\theta) / \sin \theta) \in A$. This assumption is unnecessary and will be removed at a later time [6].

6. Acknowledgement. I would like to thank Professor P. G. Nevai for many useful discussions.

Appendix A. In this appendix an alternative proof of the sufficiency part of Theorem 1.1 is given. It uses the discrete analog of the Marchenko equation and exhibits the close connection between the moment problem and discrete inverse scattering theory.

We begin again by noting that $\ln (\sigma(\theta) / \sin \theta) \in A$ and (1.8) imply via the Wiener-Levy theorem that $\ln (\sigma(\theta) / \sin \theta) \in A_{v}$. Baxter [1] has shown that $\ln (\sigma(\theta) / \sin \theta) \in A_{v}$ implies that one can have a sequence of polynomials $\{\phi(Z, n)\}$ on the unit circle orthonormal with respect to $\sigma(\theta) / \sin \theta$ such that

$$
\phi(Z, n) \in A^{+} \text {. }
$$

One can now follow the arguments leading to (3.38) to show

$$
(Z-1 / Z) p(\lambda, n) \in A
$$

where $\lambda$ is given in (3.27) and $p(\lambda, n)$ is orthonormal with respect to $\sigma(\lambda)$. Equation (A.2) and $\ln (\sigma(\theta) / \sin \theta) \in A$ imply that $a(\infty)$ and $b(\infty)$ exist. Thus one can construct $f_{+}(Z)$ such that

$$
Z f_{+}(Z) \in A_{v}^{+}, \quad Z f_{+}(Z) \neq 0 \text { for }|Z|<1,\left.Z f_{+}(Z)\right|_{z=0}>0,
$$

and

$$
\frac{\sigma(\theta)}{\sin \theta}=\frac{a(\infty)}{\pi K(0)^{2}} \frac{1}{\left|Z f_{+}(Z)\right|^{2}}
$$

Differentiating the above equation with respect to $\theta$, multiplying by $\left(1-Z^{2}\right)\left|Z f_{+}(Z)\right|^{2}$, applying $E_{+}$the operator that projects $A_{v}$ onto $A_{v}^{+}$, then multiplying by $Z f_{+}(z)$ gives

$$
\begin{aligned}
& \left(1-Z^{2}\right)\left(Z f_{+}(Z)\right)^{\prime}=-Z f_{+}(Z) E_{+}\left\{\left(1-Z^{2}\right) \frac{\left(1 / Z f_{+}(1 / Z)\right)^{\prime}}{1 / Z f_{+}(1 / Z)}\right\} \\
& +\frac{\pi K(0)^{2}}{a(\infty)} Z f_{+}(Z) E_{+}\left\{\left|Z f_{+}(Z)\right|^{2}\left(1-Z^{2}\right)\left(\frac{\sigma(\theta)}{\sin \theta}\right)^{\prime}\right\}, \quad Z=e^{i \theta}
\end{aligned}
$$

Since $1 / Z f_{+}(1 / Z)$ is an element of $A_{v}^{-}$the first term on the right-hand side has only two terms and is clearly an element $A_{0}^{+}$. Equation (1.8) and the properties of $Z f_{+}(Z)$ imply that the second term on the right-hand side belongs to $A_{v}^{+}$. Therefore

$$
\left(1-Z^{2}\right)\left(Z f_{+}(Z)\right)^{\prime} \in A_{v}^{+}, \quad Z=e^{i \theta} .
$$

Defining

$$
S(Z)=f_{+}(1 / Z) / f_{+}(Z), \quad|Z|=1
$$


it is clear that

$$
\left(1-Z^{-2}\right) S^{\prime}(Z) \in A_{v}, \quad Z=e^{i \theta},
$$

where the differentiation is with respect to $\theta$.

It is a consequence of (3.25), (A.2) and the boundary conditions that

$$
p_{+}(Z, n)=Z^{n} A(n, n)\left(1+\sum_{i=1}^{\infty} \alpha(n, i) Z^{\prime}\right) \in A^{+},
$$

and it is known that the Fourier coefficients of $p_{+}(Z, n)$ satisfy the discrete analog of the Marchenko equation [4], [8]:

$$
\omega(2 n+m)+\alpha(n, m)+\sum_{l=1}^{\infty} \alpha(n, l) \omega(l+2 n+m)=0, \quad m>n>0,
$$

where

$$
\omega(n)=\frac{1}{2 \pi} \oint(1-s(Z)) Z^{n} \frac{d Z}{Z}, \quad|Z|=1
$$

Therefore,

$$
\begin{aligned}
& \sum_{n=1}^{\infty} n v(2 n+2)|\alpha(n, m)-\alpha(n+1, m)| \\
& <\sum_{n=1}^{\infty} n v(2 n+2)|\omega(2 n+m+2)-\omega(2 n+m)| \\
& \quad+\sum_{n=1}^{\infty} n v(2 n+2)|\omega(2 n+l+m+2)-\omega(l+2 n+m)| \sum_{l=1}^{\infty}|\alpha(n+1, l)| \\
& \quad+\sum_{n=1}^{\infty} n v(2 n+2)|\alpha(n+1, l)-\alpha(n, l)| \sum_{l=1}^{\infty}|\omega(l+2 n+m)| .
\end{aligned}
$$

Since $\omega(n)$ is summable there exists $\omega_{1}(n), \hat{\omega}(n)$ and $N$ such that

$$
\begin{gathered}
\omega_{1}(n)=\omega(n)-\hat{\omega}(n), \hat{\omega}(n), \omega_{1}(n) \neq 0, \quad n<N, \\
\omega_{1}(n)=\omega(n), \quad n>N,
\end{gathered}
$$

and

$$
\sum\left|\omega_{1}(n)\right|<1 .
$$

Substituting these equations into (A.11) gives for the third term on the R.H.S. of (A.11),

$$
\begin{aligned}
& <C \sum_{n=1}^{\infty} n v(2 n+2) \\
& \quad \times\{|\omega(2 n+3)-\omega(2 n+1)|+|\omega(2 n+4)-\omega(2 n+2)|\} /\left(1-\sum_{l=1}^{\infty}\left|\omega_{1}(l)\right|\right) \\
& \quad+\frac{C \sum_{l=1}^{N} \sum_{m-1}^{N}|\hat{\omega}(l+2 n+m)| \sum_{n=1}^{N} n v(2 n+2)|\alpha(n+1, l)-\alpha(n, l)|}{1-\sum_{l=1}^{\infty}\left|\omega_{1}(l)\right|}<\infty .
\end{aligned}
$$


From the recurrence formulas one finds

$$
\alpha(n, 1)-\alpha(n-1,1)=B(n)
$$

and

$$
\alpha(n, 2)-\alpha(n-1,2)=\left(1-\frac{a(n+1)^{2}}{a(\infty)^{2}}\right)+B(n) \alpha(n, n+1) .
$$

Equations (A.11), (A.15)-(A.17), the monotonicity of $v(n)$ and the fact that the Fourier coefficients $p_{+}(Z, n)$ are bounded and summable for all $n$ give the desired result.

\section{REFERENCES}

1. G. Baxter, A convergence equivalence related to polynomials orthogonal on the unit circle, Trans. Amer. Math. Soc. 99 (1961), 471-487.

2. J. Favard, Sur les polynomes de Tchebycheff, C. R. Acad. Sci. Paris 200 (1935), 2052-2053.

3. G. Freud, Orthogonal polynomials, Pergamon Press, New York, 1971.

4. J. S. Geronimo and K. M. Case, Scattering theory and polynomials orthogonal on the real line, Trans. Amer. Math. Soc. (to appear).

5. Scattering theory and polynomials orthogonal on the unit circle, J. Mathematical Phys. 20 (1979), 299-310.

6. J. S. Geronimo and P. G. Nevai, Necessary and sufficient conditions relating the coefficients in the recurrence formula to the spectral function for orthogonal polynomials, SIAM (submitted).

7. Ya. L. Geronimus, Polynomials orthogonal on a circle and interval, (J. N. Sneddon, ed.), Pergamon Press, New York, 1960.

8. G. S. Guseinov, The determination of an infinite Jacobi matrix from the scattering data, Soviet Math. Dokl. 17 (1976), 596-600.

9. G. Szego, Orthogonal polynomials, Amer. Math. Soc. Colloq. Publ., no. 23, Amer. Math. Soc., Providence, R. I., 1939.

Georgia Institute of Technology, School of Mathematics, Atlanta, Grorgin 30332 